\title{
Pair of generalized derivations acting on multilinear polynomials in prime rings
}

\author{
Basudeb Dhara*1 (D), Sukhendu $\operatorname{Kar}^{2}$ (D), Priyadwip Das ${ }^{2}$ \\ ${ }^{1}$ Department of Mathematics, Belda College, Belda, Paschim Medinipur, 721424, W.B., India \\ ${ }^{2}$ Department of Mathematics, Jadavpur University, Kolkata-700032, W.B., India
}

\begin{abstract}
Let $R$ be a noncommutative prime ring of characteristic different from 2 with Utumi quotient ring $U$ and extended centroid $C$ and $f\left(r_{1}, \ldots, r_{n}\right)$ be a multilinear polynomial over $C$, which is not central valued on $R$. Suppose that $F$ and $G$ are two nonzero generalized derivations of $R$ such that $G \neq I d$ (identity map) and

$$
F\left(f(r)^{2}\right)=F(f(r)) G(f(r))+G(f(r)) F(f(r))
$$

for all $r=\left(r_{1}, \ldots, r_{n}\right) \in R^{n}$. Then one of the following holds:

(1) there exist $\lambda \in C$ and $\mu \in C$ such that $F(x)=\lambda x$ and $G(x)=\mu x$ for all $x \in R$ with $2 \mu=1$

(2) there exist $\lambda \in C$ and $p, q \in U$ such that $F(x)=\lambda x$ and $G(x)=p x+x q$ for all $x \in R$ with $p+q \in C, 2(p+q)=1$ and $f\left(x_{1}, \ldots, x_{n}\right)^{2}$ is central valued on $R$;

(3) there exist $\lambda \in C$ and $a \in U$ such that $F(x)=[a, x]$ and $G(x)=\lambda x$ for all $x \in R$ with $f\left(x_{1}, \ldots, x_{n}\right)^{2}$ is central valued on $R$;

(4) there exist $\lambda \in C$ and $a, b \in U$ such that $F(x)=a x+x b$ and $G(x)=\lambda x$ for all $x \in R$ with $a+b \in C, 2 \lambda=1$ and $f\left(x_{1}, \ldots, x_{n}\right)^{2}$ is central valued on $R$;

(5) there exist $a, p \in U$ such that $F(x)=x a$ and $G(x)=p x$ for all $x \in R$, with $(p-1) a=-a p \in C$ and $f\left(x_{1}, \ldots, x_{n}\right)^{2}$ is central valued on $R$;

(6) there exist $a, q \in U$ such that $F(x)=a x$ and $G(x)=x q$ for all $x \in R$ with $a(q-1)=-q a \in C$ and $f\left(x_{1}, \ldots, x_{n}\right)^{2}$ is central valued on $R$.
\end{abstract}

Mathematics Subject Classification (2010). 16N60, 16W25, 16R50

Keywords. prime ring, derivation, generalized derivation, extended centroid, Utumi quotient ring

\section{Introduction}

Throughout this paper $R$ always denotes an associative prime ring with extended centroid $C$ and $U$ its Utumi ring of quotients. By a derivation, we mean an additive mapping $d: R \rightarrow R$ such that $d(x y)=d(x) y+x d(y)$ holds for all $x, y \in R$. By a generalized derivation, we mean an additive mapping $F: R \rightarrow R$ such that $F(x y)=F(x) y+x d(y)$

\footnotetext{
*Corresponding Author.

Email addresses: basu_dhara@yahoo.com (B. Dhara), karsukhendu@yahoo.co.in (S. Kar), priyadip72@gmail.com (P. Das)

Received: 23.12.2017; Accepted: 18.03.2019
} 
holds for all $x, y \in R$, where $d$ is a derivation of $R$. Thus any derivation is a generalized derivation.

A famous result proved by Posner [17, Theorem 2] states that if a prime ring $R$ has a nonzero derivation $d$ such that $[d(x), x] \in Z(R)$ for all $x \in R$, then $R$ is commutative. Brešar [2] studied the case $d(x) x-x \delta(x) \in Z(R)$ for all $x \in R$, where $d$ and $\delta$ are two derivations of a prime ring $R$ and obtained that either $d=\delta=0$ or $R$ is commutative. After that in [13] Lee and Shiue extended the previous result considering multilinear polynomial. They proved that if $d\left(f\left(x_{1}, \ldots, x_{n}\right)\right) f\left(x_{1}, \ldots, x_{n}\right)-f\left(x_{1}, \ldots, x_{n}\right) \delta\left(f\left(x_{1}, \ldots, x_{n}\right)\right) \in Z(R)$ for all $x_{1}, \ldots, x_{n} \in I$, where $I$ is a nonzero ideal of $R$ and $f\left(x_{1}, \ldots, x_{n}\right)$ is a non-central multilinear polynomial over $C$, then either $d=0=\delta$ or $d=-\delta$ and $f\left(x_{1}, \ldots, x_{n}\right)^{2}$ is central valued on $R C$ unless $\operatorname{char}(R)=2$ and $\operatorname{dim}_{C} R C=4$.

Recently in [4], De Filippis et al. showed that if $d$ and $\delta$ are nonzero derivations of $R$ and $f\left(x_{1}, \ldots, x_{n}\right)$ is a multilinear polynomial over $C$, non-central valued on $R$, such that $\left[d\left(f\left(x_{1}, \ldots, x_{n}\right)\right), \delta\left(f\left(x_{1}, \ldots, x_{n}\right)\right)\right] \in Z(R)$ for all $x_{1}, \ldots, x_{n} \in R$, then $\{d, \delta\}$ are linear dependent over $C$ unless when $\operatorname{char}(R)=2$ and $\operatorname{dim}_{C} R C=4$.

More recently, Fosner and Vukman [7] proved that if $R$ is a prime ring of $\operatorname{char}(R) \neq 2$, $F_{1}$ and $F_{2}$ are generalized derivations of $R$ such that $F_{1}(x) F_{2}(x)+F_{2}(x) F_{1}(x)=0$ for all $x \in R$ then either $F_{1}=0$ or $F_{2}=0$. In [18], Rania and Scudo extended this result to the case $G\left(f\left(x_{1}, \ldots, x_{n}\right)\right) d\left(f\left(x_{1}, \ldots, x_{n}\right)\right)+d\left(f\left(x_{1}, \ldots, x_{n}\right)\right) G\left(f\left(x_{1}, \ldots, x_{n}\right)\right)=0$ for all $x_{1}, \ldots, x_{n} \in R$, where $G$ is a generalized derivation of $R$ and $d$ is any derivation of $R$, and proved that either $G=0$ or $d=0$, except when $d$ is inner, there exists $\lambda \in C$ such that $G(x)=\lambda x, \forall x \in R$ and $f\left(x_{1}, \ldots, x_{n}\right)^{2}$ is central valued on $R$. Recently, in [19] Yarbil and De Filippis studied the same situation, when $G$ and $d$ are two skew derivations of $R$ associated to the same automorphism $\alpha$ and obtained that either $G=0$ or $d=0$. Here skew derivation means an additive mapping $d: R \rightarrow R$ such that $d(x y)=d(x) y+\alpha(x) d(y)$ for all $x, y \in R$, where $\alpha$ is an automorphism of $R$.

Recently, Dhara et al. [6] extended the above result by taking generalized derivation $F$ in the place of derivation $d$, that is,

$$
F\left(f\left(x_{1}, \ldots, x_{n}\right)\right) G\left(f\left(x_{1}, \ldots, x_{n}\right)\right)+G\left(f\left(x_{1}, \ldots, x_{n}\right)\right) F\left(f\left(x_{1}, \ldots, x_{n}\right)\right)=0,
$$

where $F, G$ are two generalized derivations of $R$. In the present paper, we consider the case $F\left(f(r)^{2}\right)=F(f(r)) G(f(r))+G(f(r)) F(f(r))$ for all $r=\left(r_{1}, \ldots, r_{n}\right) \in R^{n}$, where $F$ and $G$ are two generalized derivations of $R$. If $G=I d$ (identity map), then $F$ becomes a derivation of $R$. So our interest is to study the case when $G \neq I d$. More precisely, we prove the following theorem.

Main Theorem. Let $R$ be a noncommutative prime ring of characteristic different from 2 with Utumi quotient ring $U$ and extended centroid $C$, and $f\left(r_{1}, \ldots, r_{n}\right)$ be a multilinear polynomial over $C$, which is not central valued on $R$. Suppose that $F$ and $G$ are two nonzero generalized derivations of $R$ such that $G \neq I d$ (identity map) and

$$
F\left(f(r)^{2}\right)=F(f(r)) G(f(r))+G(f(r)) F(f(r))
$$

for all $r=\left(r_{1}, \ldots, r_{n}\right) \in R^{n}$. Then one of the following holds:

(1) there exist $\lambda \in C$ and $\mu \in C$ such that $F(x)=\lambda x$ and $G(x)=\mu x$ for all $x \in R$ with $2 \mu=1$;

(2) there exist $\lambda \in C$ and $p, q \in U$ such that $F(x)=\lambda x$ and $G(x)=p x+x q$ for all $x \in R$ with $p+q \in C, 2(p+q)=1$, and $f\left(x_{1}, \ldots, x_{n}\right)^{2}$ is central valued on $R$;

(3) there exist $\lambda \in C$ and $a \in U$ such that $F(x)=[a, x]$ and $G(x)=\lambda x$ for all $x \in R$ with $f\left(x_{1}, \ldots, x_{n}\right)^{2}$ is central valued on $R$;

(4) there exist $\lambda \in C$ and $a, b \in U$ such that $F(x)=a x+x b$ and $G(x)=\lambda x$ for all $x \in R$ with $a+b \in C, 2 \lambda=1$ and $f\left(x_{1}, \ldots, x_{n}\right)^{2}$ is central valued on $R$; 
(5) there exist $a, p \in U$ such that $F(x)=x a$ and $G(x)=p x$ for all $x \in R$, with $(p-1) a=-a p \in C$ and $f\left(x_{1}, \ldots, x_{n}\right)^{2}$ is central valued on $R$;

(6) there exist $a, q \in U$ such that $F(x)=$ ax and $G(x)=x q$ for all $x \in R$ with $a(q-1)=-q a \in C$ and $f\left(x_{1}, \ldots, x_{n}\right)^{2}$ is central valued on $R$.

Following corollaries are straightforward.

Corollary 1.1. Let $R$ be a noncommutative prime ring of characteristic different from 2 with Utumi quotient ring $U$ and extended centroid $C$, and $f\left(r_{1}, \ldots, r_{n}\right)$ be a multilinear polynomial over $C$, which is not central valued on $R$. Suppose that $F$ is a nonzero generalized derivation of $R$ and $d$ is a nonzero derivation of $R$ such that

$$
F\left(f(r)^{2}\right)=F(f(r)) d(f(r)+d(f(r)) F(f(r)
$$

for all $r=\left(r_{1}, \ldots, r_{n}\right) \in R^{n}$. Then there exist $\lambda \in C$ and $p \in U$ such that $F(x)=\lambda x$ and $d(x)=[p, x]$ for all $x \in R$ with $f\left(x_{1}, \ldots, x_{n}\right)^{2}$ is central valued on $R$.

Corollary 1.2. Let $R$ be a noncommutative prime ring of characteristic different from 2 with Utumi quotient ring $U$ and extended centroid $C$, and $f\left(r_{1}, \ldots, r_{n}\right)$ be a multilinear polynomial over $C$, which is not central valued on $R$. Suppose that $G$ is a nonzero generalized derivation of $R$ such that

$$
G(f(r)) f(r)+f(r) G(f(r))=f(r)^{2}
$$

for all $r=\left(r_{1}, \ldots, r_{n}\right) \in R^{n}$, then one of the following holds:

(1) there exists $\mu \in C$ such that $G(x)=\mu x$ for all $x \in R$ with $2 \mu=1$;

(2) there exist $p, q \in U$ such that $G(x)=p x+x q$ for all $x \in R$ with $p+q \in C$, $2(p+q)=1$ and $f\left(x_{1}, \ldots, x_{n}\right)^{2}$ is central valued on $R$.

\section{Main results}

Lemma 2.1. [1, Lemma 3] Let $R$ be a noncommutative prime ring with Utumi quotient ring $U$ and extended centroid $C$, and $f\left(x_{1}, \ldots, x_{n}\right)$ be a multilinear polynomial over $C$, which is not central valued on $R$. Suppose that there exist $a, b, c, q \in U$ such that $(a f(r)+$ $f(r) b) f(r)-f(r)(c f(r)+f(r) q)=0$ for all $r=\left(r_{1}, \ldots, r_{n}\right) \in R^{n}$. Then one of the following holds:

(1) $a, q \in C$ and $q-a=b-c \in C$;

(2) $f\left(x_{1}, \ldots, x_{n}\right)^{2}$ is central valued on $R$ and $q-a=b-c \in C$;

(3) $\operatorname{char}(R)=2$ and $R$ satisfies $s_{4}$.

In particular, from above Lemma, we have the followings:

Lemma 2.2. Let $R$ be a noncommutative prime ring of characteristic different from 2 with Utumi quotient ring $U$ and extended centroid $C$, and $f\left(x_{1}, \ldots, x_{n}\right)$ be a multilinear polynomial over $C$, which is not central valued on $R$. Suppose that there exist $a, b, q \in U$ such that af $(r)^{2}+f(r)^{2} q+f(r) b f(r)=0$ for all $r=\left(r_{1}, \ldots, r_{n}\right) \in R^{n}$. Then one of the following holds:

(1) $a, q \in C$ and $q+a=-b \in C$;

(2) $f\left(x_{1}, \ldots, x_{n}\right)^{2}$ is central valued on $R$ and $q+a=-b \in C$;

(3) $\operatorname{char}(R)=2$ and $R$ satisfies $s_{4}$.

Lemma 2.3. [6, Corollary 2.14] Let $R$ be a prime ring of characteristic different from 2, with Utumi quotient ring $U$ and extended centroid $C$, and $f\left(x_{1}, \ldots, x_{n}\right)$ be a multilinear polynomial over $C$. Suppose that $d$ and $\delta$ are two nonzero derivations of $R$ such that

$$
d(f(r)) \delta(f(r))+\delta(f(r)) d(f(r))=0
$$

for all $r=\left(r_{1}, \ldots, r_{n}\right) \in R^{n}$. Then $f\left(x_{1}, \ldots, x_{n}\right)$ is central valued on $R$. 
Lemma 2.4. [6, Lemma 2.10] Let $R$ be a prime ring of characteristic different from 2, $U$ its Utumi quotient ring, and $C$ its extended centroid, and $f\left(x_{1}, \ldots, x_{n}\right)$ a multilinear polynomial over $C$ which is non-central valued on $R$. Suppose that $a, b, p \in U$ such that

$$
a f(r)^{2} b+f(r) p f(r)=0
$$

for all $r=\left(r_{1}, \ldots, r_{n}\right) \in R^{n}$. Then one of the following holds:

(1) $a \in C$ and $a b=-p \in C$;

(2) $b \in C$ and $a b=-p \in C$;

(3) $f\left(x_{1}, \ldots, x_{n}\right)^{2}$ is central valued on $R$ and $a b=-p \in C$.

Lemma 2.5. [5, Lemma 1.5] Let $C$ be an infinite field and $m \geq 2$. If $A_{1}, \ldots, A_{k}$ are not scalar matrices in $M_{m}(C)$ then there exists some invertible matrix $P \in M_{m}(C)$ such that any matrices $P A_{1} P^{-1}, \ldots, P A_{k} P^{-1}$ have all nonzero entries.

Proposition 2.6. Let $R=M_{m}(C)$ be the ring of all $m \times m$ matrices over the infinite field $C, f\left(x_{1}, \ldots, x_{n}\right)$ a non-central multilinear polynomial over $C$ and $a, b, p, q \in R$. If

$$
\left(a f(r)^{2}+f(r)^{2} b\right)=(a f(r)+f(r) b)(p f(r)+f(r) q)+(p f(r)+f(r) q)(a f(r)+f(r) b)
$$

for all $r=\left(r_{1}, \ldots, r_{n}\right) \in R^{n}$, then either $a$ or $p$ and either $b$ or $q$ are scalar matrices.

Proof. By our assumption, $R$ satisfies the generalized polynomial identity

$$
\begin{gathered}
\left(a f\left(r_{1}, \ldots, r_{n}\right)^{2}+f\left(r_{1}, \ldots, r_{n}\right)^{2} b\right) \\
=\left(a f\left(r_{1}, \ldots, r_{n}\right)+f\left(r_{1}, \ldots, r_{n}\right) b\right)\left(p f\left(r_{1}, \ldots, r_{n}\right)+f\left(r_{1}, \ldots, r_{n}\right) q\right) \\
+\left(p f\left(r_{1}, \ldots, r_{n}\right)+f\left(r_{1}, \ldots, r_{n}\right) q\right)\left(a f\left(r_{1}, \ldots, r_{n}\right)+f\left(r_{1}, \ldots, r_{n}\right) b\right) .
\end{gathered}
$$

We assume first that $a \notin Z(R)$ and $p \notin Z(R)$. Now we shall show that this case leads to a contradiction.

Since $a \notin Z(R)$ and $p \notin Z(R)$, by Lemma 2.5 there exists a $C$-automorphism $\phi$ of $M_{m}(C)$ such that $a_{1}=\phi(a), p_{1}=\phi(p)$ have all nonzero entries. Clearly $a_{1}, p_{1}, b_{1}=\phi(b)$ and $q_{1}=\phi(q)$ must satisfy the condition (2.1). Without loss of generality we may replace $a, b, p, q$ with $a_{1}, b_{1}, p_{1}, q_{1}$, respectively.

Here $e_{k l}$ denotes the usual matrix unit with 1 in $(k, l)$-entry and zero elsewhere. Since $f\left(x_{1}, \ldots, x_{n}\right)$ is not central, by [14] (see also [15]), there exist $u_{1}, \ldots, u_{n} \in M_{m}(C)$ and $\gamma \in$ $C-\{0\}$ such that $f\left(u_{1}, \ldots, u_{n}\right)=\gamma e_{k l}$, with $k \neq l$. Moreover, since the set $\left\{f\left(r_{1}, \ldots, r_{n}\right)\right.$ : $\left.r_{1}, \ldots, r_{n} \in M_{m}(C)\right\}$ is invariant under the action of all $C$-automorphisms of $M_{m}(C)$, then for any $i \neq j$ there exist $r_{1}, \ldots, r_{n} \in M_{m}(C)$ such that $f\left(r_{1}, \ldots, r_{n}\right)=e_{i j}$. Hence from (2.1) we have

$$
0=\left(a e_{i j}+e_{i j} b\right)\left(p e_{i j}+e_{i j} q\right)+\left(p e_{i j}+e_{i j} q\right)\left(a e_{i j}+e_{i j} b\right)
$$

and then left multiplying by $e_{i j}$, it follows $e_{i j} a e_{i j} p e_{i j}+e_{i j} p e_{i j} a e_{i j}=0$, which gives $2 a_{j i} p_{j i}=$ 0 , that is a contradiction, since $a$ and $p$ have all nonzero entries. Thus we conclude that either $a$ or $p$ is central.

Similarly, we can prove that $b$ or $q$ is central.

Therefore we conclude that either $a$ or $p$ and either $b$ or $q$ are scalar matrices.

Proposition 2.7. Let $R=M_{m}(C)$ be the ring of all matrices over the field $C$ with $\operatorname{char}(R) \neq 2$ and $f\left(x_{1}, \ldots, x_{n}\right)$ a non-central multilinear polynomial over $C$ and $a, b, p, q \in$ $R$. If

$$
\left(a f(r)^{2}+f(r)^{2} b\right)=(a f(r)+f(r) b)(p f(r)+f(r) q)+(p f(r)+f(r) q)(a f(r)+f(r) b)
$$

for all $r=\left(r_{1}, \ldots, r_{n}\right) \in R^{n}$, then either $a$ or $p$ and either $b$ or $q$ are scalar matrices.

Proof. If one assumes that $C$ is infinite, then the conclusions follow by Proposition 2.6.

Now let $C$ be finite and $K$ be an infinite field which is an extension of the field $C$. Let 
$\bar{R}=M_{m}(K) \cong R \otimes_{C} K$. Notice that the multilinear polynomial $f\left(r_{1}, \ldots, r_{n}\right)$ is central valued on $R$ if and only if it is central valued on $\bar{R}$. Consider the generalized polynomial

$$
\begin{gathered}
P\left(r_{1}, \ldots, r_{n}\right)=\left(a f\left(r_{1}, \ldots, r_{n}\right)^{2}+f\left(r_{1}, \ldots, r_{n}\right)^{2} b\right) \\
-\left(a f\left(r_{1}, \ldots, r_{n}\right)+f\left(r_{1}, \ldots, r_{n}\right) b\right)\left(p f\left(r_{1}, \ldots, r_{n}\right)+f\left(r_{1}, \ldots, r_{n}\right) q\right) \\
-\left(p f\left(r_{1}, \ldots, r_{n}\right)+f\left(r_{1}, \ldots, r_{n}\right) q\right)\left(a f\left(r_{1}, \ldots, r_{n}\right)+f\left(r_{1}, \ldots, r_{n}\right) b\right)
\end{gathered}
$$

which is a generalized polynomial identity for $R$.

Moreover, it is a multi-homogeneous of multi-degree $(2, \ldots, 2)$ in $r_{1}, \ldots, r_{n}$.

Hence the complete linearization of $P\left(r_{1}, \ldots, r_{n}\right)$ is a multilinear generalized polynomial $\Theta\left(r_{1}, \ldots, r_{n}, y_{1}, \ldots, y_{n}\right)$ in $2 n$ indeterminates, moreover

$$
\Theta\left(r_{1}, \ldots, r_{n}, r_{1}, \ldots, r_{n}\right)=2^{n} P\left(r_{1}, \ldots, r_{n}\right) .
$$

Clearly the multilinear polynomial $\Theta\left(r_{1}, \ldots, r_{n}, y_{1}, \ldots, y_{n}\right)$ is a generalized polynomial identity for $R$ and $\bar{R}$ too. Since $\operatorname{char}(C) \neq 2$ we obtain $P\left(r_{1}, \ldots, r_{n}\right)=0$ for all $r_{1}, \ldots, r_{n} \in$ $\bar{R}$ and then conclusion follows from Proposition 2.6.

In the above Proposition, replacing $b p=b^{\prime}$ and $q a=q^{\prime}$, it is straightforward to prove the following:

Corollary 2.8. Let $R=M_{m}(C)$ be the ring of all matrices over the field $C$ with char $(R) \neq$ 2 and $f\left(x_{1}, \ldots, x_{n}\right)$ a non-central multilinear polynomial over $C$ and $a, b, p, q, b^{\prime}, q^{\prime} \in R$. If

$$
\begin{gathered}
\left(a f(r)^{2}+f(r)^{2} b\right)=a f(r)(p f(r)+f(r) q)+f(r) b^{\prime} f(r)+f(r) b f(r) q \\
+p f(r)(a f(r)+f(r) b)+f(r) q^{\prime} f(r)+f(r) q f(r) b
\end{gathered}
$$

for all $r=\left(r_{1}, \ldots, r_{n}\right) \in R^{n}$, then either $a$ or $p$ and either $b$ or $q$ are scalar matrices.

Lemma 2.9. Let $R$ be a noncommutative prime ring of characteristic different from 2 with Utumi quotient ring $U$ and extended centroid $C$, and $f\left(r_{1}, \ldots, r_{n}\right)$ be a multilinear polynomial over $C$, which is not central valued on $R$. Suppose that $F$ and $G(\neq I d$, identity map) are two nonzero inner generalized derivations of $R$ such that

$$
F\left(f(r)^{2}\right)=F(f(r)) G(f(r))+G(f(r)) F(f(r))
$$

for all $r=\left(r_{1}, \ldots, r_{n}\right) \in R^{n}$. Then one of the following holds:

(1) there exist $\lambda \in C$ and $\mu \in C$ such that $F(x)=\lambda x$ and $G(x)=\mu x$ for all $x \in R$ with $2 \mu=1$;

(2) there exist $\lambda \in C$ and $p, q \in U$ such that $F(x)=\lambda x$ and $G(x)=p x+x q$ for all $x \in R$ with $p+q \in C, 2(p+q)=1$ and $f\left(x_{1}, \ldots, x_{n}\right)^{2}$ is central valued on $R$;

(3) there exist $\lambda \in C$ and $a \in U$ such that $F(x)=[a, x]$ and $G(x)=\lambda x$ for all $x \in R$ with $f\left(x_{1}, \ldots, x_{n}\right)^{2}$ is central valued on $R$;

(4) there exist $\lambda \in C$ and $a, b \in U$ such that $F(x)=a x+x b$ and $G(x)=\lambda x$ for all $x \in R$ with $a+b \in C, 2 \lambda=1$ and $f\left(x_{1}, \ldots, x_{n}\right)^{2}$ is central valued on $R$;

(5) there exist $a, p \in U$ such that $F(x)=x a$ and $G(x)=p x$ for all $x \in R$, with $(p-1) a=-a p \in C$ and $f\left(x_{1}, \ldots, x_{n}\right)^{2}$ is central valued on $R$;

(6) there exist $a, p \in U$ such that $F(x)=$ ax and $G(x)=x q$ for all $x \in R$ with $a(q-1)=-q a \in C$ and $f\left(x_{1}, \ldots, x_{n}\right)^{2}$ is central valued on $R$.

Proof. Since $F$ and $G$ are inner generalized derivations of $R$, there exist $a, b, p, q \in U$ such that $F(x)=a x+x b$ and $G(x)=p x+x q$ for all $x \in R$. Then by hypothesis, we have

$$
\begin{gathered}
h\left(r_{1}, \ldots, r_{n}\right)=\left(a f\left(r_{1}, \ldots, r_{n}\right)^{2}+f\left(r_{1}, \ldots, r_{n}\right)^{2} b\right) \\
-\left(a f\left(r_{1}, \ldots, r_{n}\right)+f\left(r_{1}, \ldots, r_{n}\right) b\right)\left(p f\left(r_{1}, \ldots, r_{n}\right)+f\left(r_{1}, \ldots, r_{n}\right) q\right) \\
-\left(p f\left(r_{1}, \ldots, r_{n}\right)+f\left(r_{1}, \ldots, r_{n}\right) q\right)\left(a f\left(r_{1}, \ldots, r_{n}\right)+f\left(r_{1}, \ldots, r_{n}\right) b\right)=0
\end{gathered}
$$


for all $r_{1}, \ldots, r_{n} \in R$. Since $R$ and $U$ satisfy the same generalized polynomial identities (GPI) (see [3]), $U$ satisfies $h\left(r_{1}, \ldots, r_{n}\right)=0$ that is

$$
\begin{gathered}
h\left(r_{1}, \ldots, r_{n}\right)=\left(a f\left(r_{1}, \ldots, r_{n}\right)^{2}+f\left(r_{1}, \ldots, r_{n}\right)^{2} b\right) \\
-\left(a f\left(r_{1}, \ldots, r_{n}\right)+f\left(r_{1}, \ldots, r_{n}\right) b\right)\left(p f\left(r_{1}, \ldots, r_{n}\right)+f\left(r_{1}, \ldots, r_{n}\right) q\right) \\
-\left(p f\left(r_{1}, \ldots, r_{n}\right)+f\left(r_{1}, \ldots, r_{n}\right) q\right)\left(a f\left(r_{1}, \ldots, r_{n}\right)+f\left(r_{1}, \ldots, r_{n}\right) b\right)=0
\end{gathered}
$$

for all $r_{1}, \ldots, r_{n} \in U$. Suppose that $h\left(r_{1}, \ldots, r_{n}\right)$ is a trivial GPI for $U$ and $C\left\{r_{1}, \ldots, r_{n}\right\}$, the free $C$-algebra in noncommuting indeterminates $r_{1}, \ldots, r_{n}$. Then, $h\left(r_{1}, \ldots, r_{n}\right)$ is zero element in $T=U *_{C} C\left\{r_{1}, \ldots, r_{n}\right\}$. This implies that $\{a, p, 1\}$ is linearly independent over $C$. Let $\alpha p+\beta a+\gamma=0$, where $\alpha, \beta, \gamma \in C$. If $\alpha=0$, then $\beta \neq 0$ and hence $a \in C$. If $\alpha \neq 0$, then $p=\lambda a+\mu$ for some $\lambda, \mu \in C$. In this case our identity reduces to

$$
\begin{gathered}
\left(a f\left(r_{1}, \ldots, r_{n}\right)^{2}+f\left(r_{1}, \ldots, r_{n}\right)^{2} b\right) \\
-\left(a f\left(r_{1}, \ldots, r_{n}\right)+f\left(r_{1}, \ldots, r_{n}\right) b\right)\left((\lambda a+\mu) f\left(r_{1}, \ldots, r_{n}\right)+f\left(r_{1}, \ldots, r_{n}\right) q\right) \\
-\left((\lambda a+\mu) f\left(r_{1}, \ldots, r_{n}\right)+f\left(r_{1}, \ldots, r_{n}\right) q\right)\left(a f\left(r_{1}, \ldots, r_{n}\right)\right. \\
\left.+f\left(r_{1}, \ldots, r_{n}\right) b\right)=0
\end{gathered}
$$

in $T$. If $a$ is not in $C$, then from above we have

$$
\begin{aligned}
& a f\left(r_{1}, \ldots, r_{n}\right)\left(\left(f\left(r_{1}, \ldots, r_{n}\right)-2 \lambda a f\left(r_{1}, \ldots, r_{n}\right)-\mu f\left(r_{1}, \ldots, r_{n}\right)\right.\right. \\
& \left.-f\left(r_{1}, \ldots, r_{n}\right) q-\lambda f\left(r_{1}, \ldots, r_{n}\right) b\right)=0
\end{aligned}
$$

in $T$, that is

$$
a f\left(r_{1}, \ldots, r_{n}\right)\left(2 \lambda a f\left(r_{1}, \ldots, r_{n}\right)+f\left(r_{1}, \ldots, r_{n}\right)(\mu+q+\lambda b-1)\right)=0 .
$$

This implies that $\lambda a \in C$ and hence $p=(\lambda a+\mu) \in C$. Thus we conclude that either $a \in C$ or $p \in C$. Similarly, we can prove that either $b \in C$ or $q \in C$.

Next suppose that $h\left(r_{1}, \ldots, r_{n}\right)$ is a non-trivial GPI for $U$. In case $C$ is infinite, we have $h\left(r_{1}, \ldots, r_{n}\right)=0$ for all $r_{1}, \ldots, r_{n} \in U \otimes_{C} \bar{C}$, where $\bar{C}$ is the algebraic closure of $C$. Since both $U$ and $U \otimes_{C} \bar{C}$ are prime and centrally closed [8, Theorems 2.5 and 3.5], we may replace $R$ by $U$ or $U \otimes_{C} \bar{C}$ according to $C$ finite or infinite. Then $R$ is centrally closed over $C$ and $h\left(r_{1}, \ldots, r_{n}\right)=0$ for all $r_{1}, \ldots, r_{n} \in R$. By Martindale's theorem [16], $R$ is then a primitive ring with nonzero socle $\operatorname{soc}(R)$ and with $C$ as its associated division ring. Then, by Jacobson's theorem [10, p.75], $R$ is isomorphic to a dense ring of linear transformations of a vector space $V$ over $C$. Assume first that $V$ is finite dimensional over $C$, that is, $\operatorname{dim}_{C} V=m$. By density of $R$, we have $R \cong M_{m}(C)$. Since $f\left(r_{1}, \ldots, r_{n}\right)$ is not central valued on $R, R$ must be noncommutative and so $m \geq 2$. In this case, by Proposition 2.7, we get that either $a$ or $p$ and either $b$ or $q$ are in $C$. If $V$ is infinite dimensional over $C$, then for any $e^{2}=e \in \operatorname{soc}(R)$ we have $e R e \cong M_{t}(C)$ with $t=\operatorname{dim}_{C} V e$. In this case we prove that either $a$ or $p$ are in $C$. To prove this, assume that $a \notin C$ and $p \notin C$. Then there exist $h_{1}, h_{2} \in \operatorname{soc}(R)$ such that $\left[a, h_{1}\right] \neq 0$ and $\left[p, h_{2}\right] \neq 0$. By Litoff's Theorem [9], there exists idempotent $e \in \operatorname{soc}(R)$ such that $a h_{1}, h_{1} a, p h_{2}, h_{2} p, h_{1}, h_{2} \in e R e$. We have $e R e \cong M_{k}(C)$ with $k=\operatorname{dim}_{C} V e$. Since $R$ satisfies generalized identity

$$
\begin{aligned}
& e\left\{a f\left(e r_{1} e, \ldots, e r_{n} e\right)^{2}+f\left(e r_{1} e, \ldots, e r_{n} e\right)^{2} b\right\} e \\
& =e\left\{\left(a f\left(e r_{1} e, \ldots, e r_{n} e\right)+f\left(e r_{1} e, \ldots, e r_{n} e\right) b\right)\right. \\
& .\left(p f\left(e r_{1} e, \ldots, e r_{n} e\right)+f\left(e r_{1} e, \ldots, e r_{n} e\right) q\right) \\
& +\left(p f\left(e r_{1} e, \ldots, e r_{n} e\right)+f\left(e r_{1} e, \ldots, e r_{n} e\right) q\right) \\
& \left..\left(a f\left(e r_{1} e, \ldots, e r_{n} e\right)+f\left(e r_{1} e, \ldots, e r_{n} e\right) b\right)\right\} e,
\end{aligned}
$$


the subring $e$ Re satisfies

$$
\begin{aligned}
& \text { eaef }\left(r_{1}, \ldots, r_{n}\right)^{2}+f\left(r_{1}, \ldots, r_{n}\right)^{2} \text { ebe } \\
& =\text { eaef }\left(r_{1}, \ldots, r_{n}\right)\left(\operatorname{epe} f\left(r_{1}, \ldots, r_{n}\right)+f\left(r_{1}, \ldots, r_{n}\right) \text { eqe }\right) \\
& +f\left(r_{1}, \ldots, r_{n}\right) \text { ebpe } f\left(r_{1}, \ldots, r_{n}\right)+f\left(r_{1}, \ldots, r_{n}\right) \text { ebe } f\left(r_{1}, \ldots, r_{n}\right) \text { eqe } \\
& + \text { epe } f\left(r_{1}, \ldots, r_{n}\right)\left(\text { eaef }\left(r_{1}, \ldots, r_{n}\right)+f\left(r_{1}, \ldots, r_{n}\right) \text { ebe }\right) \\
& +f\left(r_{1}, \ldots, r_{n}\right) \text { eqaef }\left(r_{1}, \ldots, r_{n}\right)+f\left(r_{1}, \ldots, r_{n}\right) \text { eqe }\left(r_{1}, \ldots, r_{n}\right) \text { ebe. }
\end{aligned}
$$

Then by Corollary 2.8, either eae or epe are central elements of eRe. Thus either $a h_{1}=($ eae $) h_{1}=h_{1} e a e=h_{1} a$ or $p h_{2}=($ epe $) h_{2}=h_{2}(e p e)=h_{2} p$, a contradiction. Hence either $a$ or $p$ are in $C$.

Similarly, we can prove that either $b$ or $q$ are in $C$.

Thus we have the following cases:

Case 1: Let $a, b \in C$.

In this case, by (2.5) $U$ satisfies

$$
\begin{gathered}
(a+b) f\left(r_{1}, \ldots, r_{n}\right)^{2}-(a+b)\left(f\left(r_{1}, \ldots, r_{n}\right)\left(p f\left(r_{1}, \ldots, r_{n}\right)+f\left(r_{1}, \ldots, r_{n}\right) q\right)-\right. \\
\left(p f\left(r_{1}, \ldots, r_{n}\right)+f\left(r_{1}, \ldots, r_{n}\right) q\right)(a+b) f\left(r_{1}, \ldots, r_{n}\right)=0 .
\end{gathered}
$$

Since $F \neq 0, a+b \neq 0$. Hence from above

$$
\begin{aligned}
& \left.f\left(r_{1}, \ldots, r_{n}\right)^{2}-\left(f\left(r_{1}, \ldots, r_{n}\right) p f\left(r_{1}, \ldots, r_{n}\right)+f\left(r_{1}, \ldots, r_{n}\right)^{2} q\right)\right) \\
& -\left(p f\left(r_{1}, \ldots, r_{n}\right)^{2}+f\left(r_{1}, \ldots, r_{n}\right) q f\left(r_{1}, \ldots, r_{n}\right)\right)=0 .
\end{aligned}
$$

This implies

$$
p f\left(r_{1}, \ldots, r_{n}\right)^{2}+f\left(r_{1}, \ldots, r_{n}\right)^{2}(q-1)+f\left(r_{1}, \ldots, r_{n}\right)(p+q) f\left(r_{1}, \ldots, r_{n}\right)=0,
$$

for all $r_{1}, \ldots, r_{n} \in U$. Then by Lemma 2.2 , one of the following holds:

(1) $p, q-1 \in C$ and $p+q-1=-(p+q) \in C$. In this case we have $F(x)=a x+x b=$ $(a+b) x$ and $G(x)=p x+x q=(p+q) x$ for all $x \in R$, with $2(p+q)=1$ which is our conclusion (1).

(2) $f\left(x_{1}, \ldots, x_{n}\right)^{2}$ is central valued on $R$ and $p+q-1=-(p+q) \in C$. In this case, we have $F(x)=a x+x b=(a+b) x$ and $G(x)=p x+x q$ for all $x \in R$ with $p+q \in C$ and $2(p+q)=1$, which is our conclusion (2).

Case 2: Let $p \in C$ and $q \in C$.

Then by (2.5), $U$ satisfies

$$
\begin{gathered}
\left(a f\left(r_{1}, \ldots, r_{n}\right)^{2}+f\left(r_{1}, \ldots, r_{n}\right)^{2} b\right) \\
-\left(a f\left(r_{1}, \ldots, r_{n}\right)+f\left(r_{1}, \ldots, r_{n}\right) b\right)(p+q) f\left(r_{1}, \ldots, r_{n}\right) \\
-(p+q) f\left(r_{1}, \ldots, r_{n}\right)\left(a f\left(r_{1}, \ldots, r_{n}\right)+f\left(r_{1}, \ldots, r_{n}\right) b\right)=0 .
\end{gathered}
$$

This can be written as

$$
\begin{aligned}
& a(1-p-q) f\left(r_{1}, \ldots, r_{n}\right)^{2}+f\left(r_{1}, \ldots, r_{n}\right)^{2} b(1-p-q) \\
& -\left(f\left(r_{1}, \ldots, r_{n}\right)(a+b)(p+q) f\left(r_{1}, \ldots, r_{n}\right)=0\right.
\end{aligned}
$$

for all $r_{1}, \ldots, r_{n} \in U$. Then by Lemma 2.2, one of the following holds:

(1) $a(1-p-q), b(1-p-q) \in C$ and $a(1-p-q)+b(1-p-q)=(a+b)(p+q) \in C$. Since $G \neq I d, p+q \neq 1$ and hence $a, b \in C$. Then conclusion follows by Case 1 .

(2) $f\left(x_{1}, \ldots, x_{n}\right)^{2}$ is central valued on $R$ and $a(1-p-q)+b(1-p-q)=(a+b)(p+q) \in C$. This implies $2(p+q)(a+b)=a+b$. Since $G \neq 0,0 \neq p+q \in C$. Hence $(a+b)(p+q) \in$ $C$ yields $a+b \in C$. Thus $2(p+q)(a+b)=a+b$ gives $(2(p+q)-1)(a+b)=0$. This implies either $a+b=0$ or $2(p+q)=1$. When $a+b=0, F(x)=a x+x b=[a, x]$ 
for all $x \in R, G(x)=p x+x q=(p+q) x$ for all $x \in R$, which is conclusion (3). On the other hand when $2(p+q)=1$, then $F(x)=a x+x b$ for all $x \in R$ with $a+b \in C$ and $G(x)=p x+x q=(p+q) x$ for all $x \in R$ with $2(p+q)=1$, which is our conclusion (4).

Case 3: Let $a \in C$ and $q \in C$.

Then by (2.5), we have

$$
\begin{gathered}
f\left(r_{1}, \ldots, r_{n}\right)^{2}(a+b) \\
=f\left(r_{1}, \ldots, r_{n}\right)(a+b)(p+q) f\left(r_{1}, \ldots, r_{n}\right)+(p+q) f\left(r_{1}, \ldots, r_{n}\right)^{2}(a+b),
\end{gathered}
$$

for all $r_{1}, \ldots, r_{n} \in U$.

This can be written as

$$
(p+q-1) f\left(r_{1}, \ldots, r_{n}\right)^{2}(a+b)+f\left(r_{1}, \ldots, r_{n}\right)(a+b)(p+q) f\left(r_{1}, \ldots, r_{n}\right)=0 .
$$

Then by Lemma 2.4, one of the following holds:

(1) $p+q-1,(a+b)(p+q) \in C$ and $(p+q-1)(a+b)+(a+b)(p+q)=0$. This implies $p+q \in C$. Since $G \neq 0, p+q \neq 0$ and hence $0 \neq a+b \in C$. Hence $(p+q-1)(a+b)+(a+b)(p+q)=0$ yields $2(p+q)=1$. Thus in this case we have $F(x)=a x+x b=x(a+b)=(a+b) x$ and $G(x)=p x+x q=(p+q) x$ for all $x \in R$ with $2(p+q)=1$, which is our conclusion (1).

(2) $a+b,(a+b)(p+q) \in C$ and $(p+q-1)(a+b)+(a+b)(p+q)=0$. Since $a \in C$, $a+b \in C$ yields $b \in C$. Since $F \neq 0, a+b \neq 0$ and thus $(a+b)(p+q) \in C$ implies $p+q \in C$. Hence, $(p+q-1)(a+b)+(a+b)(p+q)=0$ yields $2(p+q)=1$. Thus in this case we have $F(x)=a x+x b=x(a+b)=(a+b) x$ and $G(x)=p x+x q=(p+q) x$ for all $x \in R$ with $2(p+q)=1$, which is our conclusion (1).

(3) $f\left(x_{1}, \ldots, x_{n}\right)^{2}$ is central valued on $R$ and $(p+q-1)(a+b)=-(a+b)(p+q) \in C$. Thus in this case we have $F(x)=a x+x b=x(a+b)$ for all $x \in R$ and $G(x)=$ $p x+x q=(p+q) x$ for all $x \in R$, which is our conclusion (5).

Case 4: Let $b \in C$ and $p \in C$.

Then by (2.5), we have

$$
\begin{gathered}
(a+b) f\left(r_{1}, \ldots, r_{n}\right)^{2} \\
=(a+b) f\left(r_{1}, \ldots, r_{n}\right)^{2}(p+q)+f\left(r_{1}, \ldots, r_{n}\right)(p+q)(a+b) f\left(r_{1}, \ldots, r_{n}\right),
\end{gathered}
$$

for all $r_{1}, \ldots, r_{n} \in U$. This can be written as

$$
(a+b) f\left(r_{1}, \ldots, r_{n}\right)^{2}(p+q-1)+f\left(r_{1}, \ldots, r_{n}\right)(p+q)(a+b) f\left(r_{1}, \ldots, r_{n}\right)=0
$$

for all $r_{1}, \ldots, r_{n} \in U$. Then by Lemma 2.4, one of the following holds:

(1) $a+b,(p+q)(a+b) \in C$ and $(a+b)(p+q-1)+(p+q)(a+b)=0$. Since $b \in C$, $a+b \in C$ yields $a \in C$. Since $F \neq 0, a+b \neq 0$ and thus $(p+q)(a+b) \in C$ implies $p+q \in C$. Hence, $(a+b)(p+q-1)+(p+q)(a+b)=0$ yields $2(p+q)=1$. Thus in this case we have $F(x)=(a+b) x$ and $G(x)=(p+q) x$ for all $x \in R$ with $2(p+q)=1$, which is our conclusion (1).

(2) $p+q-1,(p+q)(a+b) \in C$ and $(a+b)(p+q-1)+(p+q)(a+b)=0$. Since $p \in C$, $p+q-1 \in C$ yields $q \in C$. Since $G \neq 0, p+q \neq 0$ and thus $(p+q)(a+b) \in C$ implies $a+b \in C$. Hence, $(a+b)(p+q-1)+(p+q)(a+b)=0$ yields $2(p+q)=1$. Thus in this case we have $F(x)=(a+b) x$ and $G(x)=(p+q) x$ for all $x \in R$ with $2(p+q)=1$, which is our conclusion (1).

(3) $f\left(x_{1}, \ldots, x_{n}\right)^{2}$ is central valued on $R$ and $(a+b)(p+q-1)=-(p+q)(a+b) \in C$. Thus in this case we have $F(x)=a x+x b=(a+b) x$ for all $x \in R$ and $G(x)=$ $p x+x q=x(p+q)$ for all $x \in R$, which is our conclusion (6). 
Proof of the Main Theorem. In [12, Theorem 3], Lee proved that every generalized derivation $g$ on a dense right ideal of $R$ can be uniquely extended to a generalized derivation of $U$ and thus can be assumed to be defined on the whole $U$ with the form $g(x)=$ $a x+d(x)$ for some $a \in U$ and $d$ is a derivation of $U$. In the light of this, we may assume that there exist $a, b \in U$ and derivations $d, \delta$ of $U$ such that $F(x)=a x+d(x)$ and $G(x)=b x+\delta(x)$. Since $I, R$, and $U$ satisfy the same generalized polynomial identities (see [3]) as well as the same differential identities (see [14]), without loss of generality, to prove our results, we may assume $F\left(f\left(x_{1}, \ldots, x_{n}\right)\right)^{2}=F\left(f\left(x_{1}, \ldots, x_{n}\right)\right) G\left(f\left(x_{1}, \ldots, x_{n}\right)\right)+$ $G\left(f\left(x_{1}, \ldots, x_{n}\right)\right) F\left(f\left(x_{1}, \ldots, x_{n}\right)\right)$ for all $x_{1}, \ldots, x_{n} \in U$.

If $F$ and $G$ both are inner generalized derivations of $R$, then by Lemma 2.9 we obtain our conclusions. Thus we assume that not both of $F$ and $G$ are inner. Hence $U$ satisfies

$$
\begin{gathered}
a f\left(x_{1}, \ldots, x_{n}\right)^{2}+d\left(f\left(x_{1}, \ldots, x_{n}\right)^{2}\right) \\
=\left(a f\left(x_{1}, \ldots, x_{n}\right)+d\left(f\left(x_{1}, \ldots, x_{n}\right)\right)\right)\left(b f\left(x_{1}, \ldots, x_{n}\right)+\delta\left(f\left(x_{1}, \ldots, x_{n}\right)\right)\right) \\
+\left(b f\left(x_{1}, \ldots, x_{n}\right)+\delta\left(f\left(x_{1}, \ldots, x_{n}\right)\right)\right)\left(a f\left(x_{1}, \ldots, x_{n}\right)+d\left(f\left(x_{1}, \ldots, x_{n}\right)\right)\right)
\end{gathered}
$$

for all $\left(x_{1}, \ldots, x_{n} \in U\right.$, where $d, \delta$ are two derivations on $U$ not both are inner.

Case 1: Assume that $d$ and $\delta$ are $C$-dependent modulo inner derivations of $U$ i.e., $\alpha d+\beta \delta=a d_{q}$, where $\alpha, \beta \in C$.

Subcase 1.i: Suppose $\alpha=0$. Then $\delta(x)=[p, x]$, where $p=\beta^{-1} q$. Obviously $d$ is not an inner derivation of $U$. From (2.16) we obtain that $U$ satisfies

$$
\begin{aligned}
& a f\left(x_{1}, \ldots, x_{n}\right)^{2}+d\left(f\left(x_{1}, \ldots, x_{n}\right)\right) f\left(x_{1}, \ldots, x_{n}\right)+f\left(x_{1}, \ldots, x_{n}\right) d\left(f\left(x_{1}, \ldots, x_{n}\right)\right) \\
& \quad=\left(a f\left(x_{1}, \ldots, x_{n}\right)+d\left(f\left(x_{1}, \ldots, x_{n}\right)\right)\right)\left(b f\left(x_{1}, \ldots, x_{n}\right)+\left[p, f\left(x_{1}, \ldots, x_{n}\right)\right]\right) \\
& \quad+\left(b f\left(x_{1}, \ldots, x_{n}\right)+\left[p, f\left(x_{1}, \ldots, x_{n}\right)\right]\right)\left(a f\left(x_{1}, \ldots, x_{n}\right)+d\left(f\left(x_{1}, \ldots, x_{n}\right)\right) .\right.
\end{aligned}
$$

Let $f^{d}\left(x_{1}, \ldots, x_{n}\right)$ be the polynomials obtained from $f\left(x_{1}, \ldots, x_{n}\right)$ replacing each coefficients $\alpha_{\sigma}$ with $d\left(\alpha_{\sigma}\right)$. Then we have

$$
d\left(f\left(x_{1}, \ldots, x_{n}\right)\right)=f^{d}\left(x_{1}, \ldots, x_{n}\right)+\sum_{i} f\left(x_{1}, \ldots, d\left(x_{i}\right), \ldots, x_{n}\right) .
$$

Thus (2.17) gives

$$
\begin{gathered}
a f\left(x_{1}, \ldots, x_{n}\right)^{2}+\left(f^{d}\left(x_{1}, \ldots, x_{n}\right)+\sum_{i} f\left(x_{1}, \ldots, d\left(x_{i}\right), \ldots, x_{n}\right)\right) f\left(x_{1}, \ldots, x_{n}\right) \\
+f\left(x_{1}, \ldots, x_{n}\right)\left(f^{d}\left(x_{1}, \ldots, x_{n}\right)+\sum_{i} f\left(x_{1}, \ldots, d\left(x_{i}\right), \ldots, x_{n}\right)\right) \\
=\left(a f\left(x_{1}, \ldots, x_{n}\right)+f^{d}\left(x_{1}, \ldots, x_{n}\right)+\sum_{i} f\left(x_{1}, \ldots, d\left(x_{i}\right), \ldots, x_{n}\right)\right) \\
\cdot\left(b f\left(x_{1}, \ldots, x_{n}\right)+\left[p, f\left(x_{1}, \ldots, x_{n}\right)\right]\right)+\left(b f\left(x_{1}, \ldots, x_{n}\right)+\left[p, f\left(x_{1}, \ldots, x_{n}\right)\right]\right) \\
\cdot\left(a f\left(x_{1}, \ldots, x_{n}\right)+f^{d}\left(x_{1}, \ldots, x_{n}\right)+\sum_{i} f\left(x_{1}, \ldots, d\left(x_{i}\right), \ldots, x_{n}\right)\right) .
\end{gathered}
$$

Since $d$ is outer derivation, by Kharchenko's theorem [11], we have that $U$ satisfies 


$$
\begin{aligned}
a f\left(x_{1}, \ldots, x_{n}\right)^{2}+\left(f^{d}\left(x_{1}, \ldots, x_{n}\right)+\sum_{i} f\left(x_{1}, \ldots, y_{i}, \ldots, x_{n}\right)\right) f\left(x_{1}, \ldots, x_{n}\right) \\
+f\left(x_{1}, \ldots, x_{n}\right)\left(\left(f^{d}\left(x_{1}, \ldots, x_{n}\right)+\sum_{i} f\left(x_{1}, \ldots, y_{i}, \ldots, x_{n}\right)\right)\right. \\
=\left(a f\left(x_{1}, \ldots, x_{n}\right)+f^{d}\left(x_{1}, \ldots, x_{n}\right)+\sum_{i} f\left(x_{1}, \ldots, y_{i}, \ldots, x_{n}\right)\right) \\
\cdot\left(b f\left(x_{1}, \ldots, x_{n}\right)+\left[p, f\left(x_{1}, \ldots, x_{n}\right)\right]\right)+\left(b f\left(x_{1}, \ldots, x_{n}\right)+\left[p, f\left(x_{1}, \ldots, x_{n}\right)\right]\right) \\
\cdot\left(a f\left(x_{1}, \ldots, x_{n}\right)+f^{d}\left(x_{1}, \ldots, x_{n}\right)+\sum_{i} f\left(x_{1}, \ldots, y_{i}, \ldots, x_{n}\right)\right) .
\end{aligned}
$$

Particularly, $U$ satisfies the blended component,

$$
\begin{aligned}
\sum_{i} f\left(x_{1}, \ldots, y_{i}, \ldots, x_{n}\right) f\left(x_{1}, \ldots, x_{n}\right)+f\left(x_{1}, \ldots, x_{n}\right) \sum_{i} f\left(x_{1}, \ldots, y_{i}, \ldots, x_{n}\right) \\
\quad=\sum_{i} f\left(x_{1}, \ldots, y_{i}, \ldots, x_{n}\right)\left(b f\left(x_{1}, \ldots, x_{n}\right)+\left[p, f\left(x_{1}, \ldots, x_{n}\right)\right]\right) \\
+\left(b f\left(x_{1}, \ldots, x_{n}\right)+\left[p, f\left(x_{1}, \ldots, x_{n}\right)\right]\right) \sum_{i} f\left(x_{1}, \ldots, y_{i}, \ldots, x_{n}\right) .
\end{aligned}
$$

In particular, for $y_{1}=x_{1}, y_{2}=y_{3}=\ldots=y_{n}=0$, we get from above

$$
\begin{aligned}
& 2 f\left(x_{1}, \ldots, x_{n}\right)^{2}=f\left(x_{1}, \ldots, x_{n}\right)\left(b f\left(x_{1}, \ldots, x_{n}\right)+\left[p, f\left(x_{1}, \ldots, x_{n}\right)\right]\right) \\
& +\left(b f\left(x_{1}, \ldots, x_{n}\right)+\left[p, f\left(x_{1}, \ldots, x_{n}\right)\right]\right) f\left(x_{1}, \ldots, x_{n}\right)
\end{aligned}
$$

which gives

$$
(b+p) f\left(x_{1}, \ldots, x_{n}\right)^{2}-f\left(x_{1}, \ldots, x_{n}\right)^{2}(p+2)+f\left(x_{1}, \ldots, x_{n}\right) b f\left(x_{1}, \ldots, x_{n}\right)=0 .
$$

Then by Lemma 2.2, one of the following holds:

(1) $b+p, p+2, b \in C$ and $(b+p)-(p+2)=-b$. This implies $p \in C$ and $b=1$. Thus in this case we have $G(x)=b x+[p, x]=x$ for all $x \in R$, a contradiction.

(2) $f\left(x_{1}, \ldots, x_{n}\right)^{2}$ is central valued on $R$ and $(b+p)-(p+2)=-b \in C$. This gives $b=1$. In this case, we have from (2.17) that $U$ satisfies

$$
\begin{gathered}
a f\left(x_{1}, \ldots, x_{n}\right)^{2}+d\left(f\left(x_{1}, \ldots, x_{n}\right)^{2}\right) \\
=\left(a f\left(x_{1}, \ldots, x_{n}\right)+d\left(f\left(x_{1}, \ldots, x_{n}\right)\right)\right)\left(f\left(x_{1}, \ldots, x_{n}\right)+\left[p, f\left(x_{1}, \ldots, x_{n}\right)\right]\right) \\
+\left(f\left(x_{1}, \ldots, x_{n}\right)+\left[p, f\left(x_{1}, \ldots, x_{n}\right)\right]\right)\left(a f\left(x_{1}, \ldots, x_{n}\right)\right. \\
\left.+d\left(f\left(x_{1}, \ldots, x_{n}\right)\right)\right) .
\end{gathered}
$$

This implies

$$
\begin{aligned}
& 0=a f\left(x_{1}, \ldots, x_{n}\right)\left[p, f\left(x_{1}, \ldots, x_{n}\right)\right]+d\left(f\left(x_{1}, \ldots, x_{n}\right)\right)\left[p, f\left(x_{1}, \ldots, x_{n}\right)\right] \\
& +f\left(x_{1}, \ldots, x_{n}\right) a f\left(x_{1}, \ldots, x_{n}\right)+\left[p, f\left(x_{1}, \ldots, x_{n}\right)\right] a f\left(x_{1}, \ldots, x_{n}\right) \\
& +\left[p, f\left(x_{1}, \ldots, x_{n}\right)\right] d\left(\left(f\left(x_{1}, \ldots, x_{n}\right)\right) .\right.
\end{aligned}
$$

It gives

$$
\begin{gathered}
0=a f\left(x_{1}, \ldots, x_{n}\right)\left[p, f\left(x_{1}, \ldots, x_{n}\right)\right] \\
+\left(f^{d}\left(x_{1}, \ldots, x_{n}\right)+\sum_{i} f\left(x_{1}, \ldots, y_{i}, \ldots, x_{n}\right)\right)\left[p, f\left(x_{1}, \ldots, x_{n}\right)\right] \\
+f\left(x_{1}, \ldots, x_{n}\right) a f\left(x_{1}, \ldots, x_{n}\right)+\left[p, f\left(x_{1}, \ldots, x_{n}\right)\right] a f\left(x_{1}, \ldots, x_{n}\right) \\
+\left[p, f\left(x_{1}, \ldots, x_{n}\right)\right]\left(f^{d}\left(x_{1}, \ldots, x_{n}\right)+\sum_{i} f\left(x_{1}, \ldots, y_{i}, \ldots, x_{n}\right)\right) .
\end{gathered}
$$


In particular, $U$ satisfies the blended component

$$
\begin{aligned}
& \sum_{i} f\left(x_{1}, \ldots, y_{i}, \ldots, x_{n}\right)\left[p, f\left(x_{1}, \ldots, x_{n}\right)\right] \\
& +\left[p, f\left(x_{1}, \ldots, x_{n}\right)\right] \sum_{i} f\left(x_{1}, \ldots, y_{i}, \ldots, x_{n}\right)=0 .
\end{aligned}
$$

Putting $y_{i}=\left[q, x_{i}\right]$ in (2.23), where $q \notin C$, we have that $U$ satisfies

$$
\left[q, f\left(x_{1}, \ldots, x_{n}\right)\right]\left[p, f\left(x_{1}, \ldots, x_{n}\right)\right]+\left[p, f\left(x_{1}, \ldots, x_{n}\right)\right]\left[q, f\left(x_{1}, \ldots, x_{n}\right)\right]=0 .
$$

Then by Lemma 2.3, $p \in C$. Thus $G(x)=b x+[p, x]=x$ for all $x \in R$, a contradiction.

Subcase 1.ii: Suppose $\alpha \neq 0$, then $\alpha d+\beta \delta=a d_{q}$ gives $d=\mu \delta+a d_{c}$ for some $\mu \in C$ and $c \in U$. Then we can assume that $\delta$ is not an inner derivation, otherwise $d$ and $\delta$ both will be inner derivations, a contradiction. From (2.16), $U$ satisfies

$$
\begin{gathered}
a f\left(x_{1}, \ldots, x_{n}\right)^{2}+\mu \delta\left(f\left(x_{1}, \ldots, x_{n}\right)^{2}\right)+\left[c, f\left(x_{1}, \ldots, x_{n}\right)^{2}\right] \\
=\left(a f\left(x_{1}, \ldots, x_{n}\right)+\mu \delta\left(f\left(x_{1}, \ldots, x_{n}\right)\right)+\left[c, f\left(x_{1}, \ldots, x_{n}\right)\right]\right) \\
\cdot\left(b f\left(x_{1}, \ldots, x_{n}\right)+\delta\left(f\left(x_{1}, \ldots, x_{n}\right)\right)\right) \\
+\left(b f\left(x_{1}, \ldots, x_{n}\right)+\delta\left(f\left(x_{1}, \ldots, x_{n}\right)\right)\right) \\
\cdot\left(a f\left(x_{1}, \ldots, x_{n}\right)+\mu \delta\left(f\left(x_{1}, \ldots, x_{n}\right)\right)\right. \\
\left.+\left[c, f\left(x_{1}, \ldots, x_{n}\right)\right]\right),
\end{gathered}
$$

that is,

$$
\begin{gathered}
a f\left(x_{1}, \ldots, x_{n}\right)^{2}+\mu\left(f^{\delta}\left(x_{1}, \ldots, x_{n}\right)+\sum_{i} f\left(x_{1}, \ldots, \delta\left(x_{i}\right), \ldots, x_{n}\right)\right) f\left(x_{1}, \ldots, x_{n}\right) \\
+\mu f\left(x_{1}, \ldots, x_{n}\right)\left(f^{\delta}\left(x_{1}, \ldots, x_{n}\right)+\sum_{i} f\left(x_{1}, \ldots, \delta\left(x_{i}\right), \ldots, x_{n}\right)\right)+\left[c, f\left(x_{1}, \ldots, x_{n}\right)^{2}\right] \\
=\left(a f\left(x_{1}, \ldots, x_{n}\right)+\mu\left(f^{\delta}\left(x_{1}, \ldots, x_{n}\right)+\sum_{i} f\left(x_{1}, \ldots, \delta\left(x_{i}\right), \ldots, x_{n}\right)\right)+\left[c, f\left(x_{1}, \ldots, x_{n}\right)\right]\right) \\
\cdot\left(b f\left(x_{1}, \ldots, x_{n}\right)+f^{\delta}\left(x_{1}, \ldots, x_{n}\right)+\sum_{i} f\left(x_{1}, \ldots, \delta\left(x_{i}\right), \ldots, x_{n}\right)\right) \\
+\left(b f\left(x_{1}, \ldots, x_{n}\right)+\left(f^{\delta}\left(x_{1}, \ldots, x_{n}\right)+\sum_{i} f\left(x_{1}, \ldots, \delta\left(x_{i}\right), \ldots, x_{n}\right)\right)\right) \\
\cdot\left(a f\left(x_{1}, \ldots, x_{n}\right)+\mu\left(f^{\delta}\left(x_{1}, \ldots, x_{n}\right)+\sum_{i} f\left(x_{1}, \ldots, \delta\left(x_{i}\right), \ldots, x_{n}\right)\right)\right. \\
\left.+\left[c, f\left(x_{1}, \ldots, x_{n}\right)\right]\right) .
\end{gathered}
$$


Then by Kharchenko's theorem [11], we have that $U$ satisfies

$$
\begin{gathered}
a f\left(x_{1}, \ldots, x_{n}\right)^{2}+\mu\left(f^{\delta}\left(x_{1}, \ldots, x_{n}\right)+\sum_{i} f\left(x_{1}, \ldots, y_{i}, \ldots, x_{n}\right)\right) f\left(x_{1}, \ldots, x_{n}\right) \\
+\mu f\left(x_{1}, \ldots, x_{n}\right)\left(f^{\delta}\left(x_{1}, \ldots, x_{n}\right)+\sum_{i} f\left(x_{1}, \ldots, y_{i}, \ldots, x_{n}\right)\right)+\left[c, f\left(x_{1}, \ldots, x_{n}\right)^{2}\right] \\
=\left(a f\left(x_{1}, \ldots, x_{n}\right)+\mu\left(f^{\delta}\left(x_{1}, \ldots, x_{n}\right)+\sum_{i} f\left(x_{1}, \ldots, y_{i}, \ldots, x_{n}\right)\right)+\left[c, f\left(x_{1}, \ldots, x_{n}\right)\right]\right) \\
\cdot\left(b f\left(x_{1}, \ldots, x_{n}\right)+f^{\delta}\left(x_{1}, \ldots, x_{n}\right)+\sum_{i} f\left(x_{1}, \ldots, y_{i}, \ldots, x_{n}\right)\right) \\
+\left(b f\left(x_{1}, \ldots, x_{n}\right)+\left(f^{\delta}\left(x_{1}, \ldots, x_{n}\right)+\sum_{i} f\left(x_{1}, \ldots, y_{i}, \ldots, x_{n}\right)\right)\right) \\
\cdot\left(a f\left(x_{1}, \ldots, x_{n}\right)+\mu\left(f^{\delta}\left(x_{1}, \ldots, x_{n}\right)+\sum_{i} f\left(x_{1}, \ldots, y_{i}, \ldots, x_{n}\right)\right)\right. \\
\left.+\left[c, f\left(x_{1}, \ldots, x_{n}\right)\right]\right)
\end{gathered}
$$

In particular, for $x_{1}=0, U$ satisfies

$$
0=\mu f\left(x_{1}, \ldots, x_{n}\right)^{2}+\mu f\left(x_{1}, \ldots, x_{n}\right)^{2},
$$

that is, $2 \mu f\left(x_{1}, \ldots, x_{n}\right)^{2}=0$. Since $\operatorname{char}(R) \neq 2, U$ satisfies $\mu f\left(x_{1}, \ldots, x_{n}\right)^{2}=0$. This implies that either $\mu=0$ or $f\left(x_{1}, \ldots, x_{n}\right)^{2}=0$. Now $f\left(x_{1}, \ldots, x_{n}\right)^{2}=0$, implies $f\left(x_{1}, \ldots, x_{n}\right)=0$ for all $x_{1}, \ldots, x_{n} \in U$, a contradiction. Hence we have $\mu=0$. Thus (2.25) reduces to

$$
\begin{gathered}
a f\left(x_{1}, \ldots, x_{n}\right)^{2}=\left(a f\left(x_{1}, \ldots, x_{n}\right)+\left[c, f\left(x_{1}, \ldots, x_{n}\right)\right]\right) \\
\cdot\left(b f\left(x_{1}, \ldots, x_{n}\right)+f^{\delta}\left(x_{1}, \ldots, x_{n}\right)+\sum_{i} f\left(x_{1}, \ldots, y_{i}, \ldots, x_{n}\right)\right) \\
+\left(b f\left(x_{1}, \ldots, x_{n}\right)+\left(f^{\delta}\left(x_{1}, \ldots, x_{n}\right)+\sum_{i} f\left(x_{1}, \ldots, y_{i}, \ldots, x_{n}\right)\right)\right) \\
\cdot\left(a f\left(x_{1}, \ldots, x_{n}\right)+\left[c, f\left(x_{1}, \ldots, x_{n}\right)\right]\right) .
\end{gathered}
$$

In particular, $U$ satisfies blended components

$$
\begin{gathered}
\left(a f\left(x_{1}, \ldots, x_{n}\right)+\left[c, f\left(x_{1}, \ldots, x_{n}\right)\right]\right) \sum_{i} f\left(x_{1}, \ldots, y_{i}, \ldots, x_{n}\right) \\
+\sum_{i} f\left(x_{1}, \ldots, y_{i}, \ldots, x_{n}\right)\left(a f\left(x_{1}, \ldots, x_{n}\right)+\left[c, f\left(x_{1}, \ldots, x_{n}\right)\right]\right)=0 .
\end{gathered}
$$

For $y_{1}=x_{1}$ and $y_{2}=y_{3}=, \ldots,=y_{n}=0, U$ satisfies

$$
\begin{gathered}
\left(a f\left(x_{1}, \ldots, x_{n}\right)+\left[c, f\left(x_{1}, \ldots, x_{n}\right)\right]\right) f\left(x_{1}, \ldots, x_{n}\right) \\
+f\left(x_{1}, \ldots, x_{n}\right)\left(a f\left(x_{1}, \ldots, x_{n}\right)+\left[c, f\left(x_{1}, \ldots, x_{n}\right)\right]\right)=0,
\end{gathered}
$$

that is

$$
(a+c) f\left(x_{1}, \ldots, x_{n}\right)^{2}-f\left(x_{1}, \ldots, x_{n}\right)^{2} c+f\left(x_{1}, \ldots, x_{n}\right) a f\left(x_{1}, \ldots, x_{n}\right)=0,
$$

for all $x_{1}, \ldots, x_{n} \in U$. Then by Lemma 2.2 , we have one of the followings:

(1) $a+c, c, a \in C$ and $2 a=0$. Thus $a=0$. In this case $F(x)=a x+[c, x]=0$ for all $x \in U$, a contradiction. 
(2) $f\left(x_{1}, \ldots, x_{n}\right)^{2}$ is central valued on $R$ and $a \in C$ with $2 a=0$. This implies $a=0$. Then by (2.27), $U$ satisfies

$$
\begin{aligned}
& {\left[c, f\left(x_{1}, \ldots, x_{n}\right)\right] \sum_{i} f\left(x_{1}, \ldots, y_{i}, \ldots, x_{n}\right)} \\
& +\sum_{i} f\left(x_{1}, \ldots, y_{i}, \ldots, x_{n}\right)\left[c, f\left(x_{1}, \ldots, x_{n}\right)\right]=0 .
\end{aligned}
$$

Replacing $y_{i}$ with $\left[q, x_{i}\right]$ for some $q \notin C$, we get from above that $U$ satisfies

$\left[c, f\left(x_{1}, \ldots, x_{n}\right)\right]\left[q, f\left(x_{1}, \ldots, x_{n}\right)\right]+\left[q, f\left(x_{1}, \ldots, x_{n}\right)\right]\left[c, f\left(x_{1}, \ldots, x_{n}\right)\right]=0$.

By Lemma 2.3, $c \in C$. Then $F(x)=a x+[c, x]=0$ for all $x \in R$, a contradiction.

Case 2: Let $d$ and $\delta$ be linearly $C$-independent modulo inner derivations of $U$. Then from (2.16), $U$ satisfies

$$
\begin{aligned}
& a f\left(x_{1}, \ldots, x_{n}\right)^{2}+\left(f^{d}\left(x_{1}, \ldots, x_{n}\right)+\sum_{i} f\left(x_{1}, \ldots, d\left(x_{i}\right), \ldots, x_{n}\right)\right) f\left(x_{1}, \ldots, x_{n}\right) \\
&+ f\left(x_{1}, \ldots, x_{n}\right)\left(f^{d}\left(x_{1}, \ldots, x_{n}\right)+\sum_{i} f\left(x_{1}, \ldots, d\left(x_{i}\right), \ldots, x_{n}\right)\right) \\
&=\left(a f\left(x_{1}, \ldots, x_{n}\right)+f^{d}\left(x_{1}, \ldots, x_{n}\right)+\sum_{i} f\left(x_{1}, \ldots, d\left(x_{i}\right), \ldots, x_{n}\right)\right) \\
& \cdot\left(b f\left(x_{1}, \ldots, x_{n}\right)+f^{\delta}\left(x_{1}, \ldots, x_{n}\right)+\sum_{i} f\left(x_{1}, \ldots, \delta\left(x_{i}\right), \ldots, x_{n}\right)\right) \\
&+\left(b f\left(x_{1}, \ldots, x_{n}\right)+f^{\delta}\left(x_{1}, \ldots, x_{n}\right)+\sum_{i} f\left(x_{1}, \ldots, \delta\left(x_{i}\right), \ldots, x_{n}\right)\right) \\
& \cdot\left(a f\left(x_{1}, \ldots, x_{n}\right)+\left(f^{d}\left(x_{1}, \ldots, x_{n}\right)+\sum_{i} f\left(x_{1}, \ldots, d\left(x_{i}\right), \ldots, x_{n}\right)\right)\right.
\end{aligned}
$$

for all $x_{1}, \ldots, x_{n} \in U$. Since $d$ and $\delta$ are not inner, by Kharchenko's theorem [11], $U$ satisfies

$$
\begin{aligned}
a f\left(x_{1}, \ldots, x_{n}\right)^{2}+\left(f^{d}\left(x_{1}, \ldots, x_{n}\right)+\sum_{i} f\left(x_{1}, \ldots, y_{i}, \ldots, x_{n}\right)\right) f\left(x_{1}, \ldots, x_{n}\right) \\
\quad+f\left(x_{1}, \ldots, x_{n}\right)\left(f^{d}\left(x_{1}, \ldots, x_{n}\right)+\sum_{i} f\left(x_{1}, \ldots, y_{i}, \ldots, x_{n}\right)\right) \\
=\left(a f\left(x_{1}, \ldots, x_{n}\right)+f^{d}\left(x_{1}, \ldots, x_{n}\right)+\sum_{i} f\left(x_{1}, \ldots, y_{i}, \ldots, x_{n}\right)\right) \\
\quad \cdot\left(b f\left(x_{1}, \ldots, x_{n}\right)+f^{\delta}\left(x_{1}, \ldots, x_{n}\right)+\sum_{i} f\left(x_{1}, \ldots, z_{i}, \ldots, x_{n}\right)\right) \\
+\left(b f\left(x_{1}, \ldots, x_{n}\right)+f^{\delta}\left(x_{1}, \ldots, x_{n}\right)+\sum_{i} f\left(x_{1}, \ldots, z_{i}, \ldots, x_{n}\right)\right) \\
\quad\left(a f\left(x_{1}, \ldots, x_{n}\right)+f^{d}\left(x_{1}, \ldots, x_{n}\right)+\sum_{i} f\left(x_{1}, \ldots, y_{i}, \ldots, x_{n}\right)\right) .
\end{aligned}
$$

In particular, for $x_{1}=0, z_{1}=y_{1}$, we get $2 f\left(y_{1}, x_{2}, \ldots, x_{n}\right)^{2}=0$ implying $f\left(x_{1}, \ldots, x_{n}\right)^{2}=$ 0 for all $x_{1}, \ldots, x_{n} \in U$. It yields $f\left(x_{1}, \ldots, x_{n}\right)=0$ for all $x_{1}, \ldots, x_{n} \in U$, a contradiction. Thus the proof of the theorem is completed.

Acknowledgment. The authors would like to thank the referee for pointing out some misprints and providing very helpful comments and suggestions. The third author expresses his thanks to the University Grants Commission, New Delhi for its JRF awarded to him under grant No. 424222 dated 22.03.2017. 


\section{References}

[1] N. Argac and V. De Filippis, Actions of generalized derivations on multilinear polynomials in prime rings, Algebra Colloq. 18 (Spec 01), 955-964, 2011.

[2] M. Brešar, Centralizing mappings and derivations in prime rings, J. Algebra, 156, 385-394, 1993.

[3] C.L. Chuang, GPIs having coefficients in Utumi quotient rings, Proc. Amer. Math. Soc. 103 (3), 723-728, 1988.

[4] V. De Filippis, O.M. Di Vincenzo, and C.Y. Pan, Quadratic central differential identities on a multilinear polynomial, Comm. Algebra, 36 (10), 3671-3681, 2008.

[5] V. De Filippis and O.M. Di Vincenzo, Vanishing derivations and centralizers of generalized derivations on multilinear polynomials, Comm. Algebra, 40, 1918-1932, 2012.

[6] B. Dhara, S. Kar, and K.G. Pradhan, Identities with generalized derivations on multilinear polynomials in prime rings, Afr. Mat. 27, 1347-1360, 2016.

[7] M. Fosner and J. Vukman, Identities with generalized derivations in prime rings, Mediter. J. Math. 9 (4), 847-863, 2012.

[8] T.S. Erickson, W.S. Martindale III, and J.M. Osborn, Prime nonassociative algebras, Pacific J. Math. 60, 49-63, 1975.

[9] C. Faith and Y. Utumi, On a new proof of Litoff's theorem, Acta Math. Acad. Sci. Hung. 14, 369-371, 1963.

[10] N. Jacobson, Structure of rings, Amer. Math. Soc. Colloq. Pub. 37, Amer. Math. Soc., Providence, RI, 1964.

[11] V.K. Kharchenko, Differential identity of prime rings, Algebra Logic, 17, 155-168, 1978.

[12] T.K. Lee, Generalized derivations of left faithful rings, Comm. Algebra, 27 (8), 40574073, 1999.

[13] T.K. Lee and W.K. Shiue, Derivations co-centralizing polynomials, Taiwanese J. Math. 2 (4), 457-467, 1998.

[14] T.K. Lee, Semiprime rings with differential identities, Bull. Inst. Math. Acad. Sinica, 20 (1), 27-38, 1992.

[15] U. Leron, Nil and power central polynomials in rings, Trans. Amer. Math. Soc. 202, 297-103, 1975.

[16] W.S. Martindale III, Prime rings satisfying a generalized polynomial identity, J. Algebra, 12, 576-584, 1969.

[17] E.C. Posner, Derivations in prime rings, Proc. Amer. Math. Soc. 8, 1093-1100, 1957.

[18] F. Rania and G. Scudo, A quadratic differential identity with generalized derivations on multilinear polynomials in prime rings, Mediterr. J. Math. 11, 273-285, 2014.

[19] N.B. Yarbil and V. De Filippis, A quadratic differential identity with skew derivations, Comm. Algebra, 46 (1), 205-216, 2018. 